Desinfektion genügend präzisiert, so ergeben sich seine Erfordernisse aus sich selbst heraus.

Um eine fast sofortige Dampfentwicklung $\mathbf{z u}$ erhalten, um jedes überflüssige Erwärmen von Wassermassen zu vermeiden, um mühelos eine geringe Uberhitzung des Dampfes zu erzielen, verfährt man am einfachsten so, daß man das Dampfentwicklungsgefäß recht klein gestaltet. Da man sich aber damit der Gefahr aussetzen würde, daß der Apparat während der Sterilisation versagen könnte, muß man, um die, durch das kleine Entwicklungsgefäß sich ergebenden Nachteile zu kompensieren, am besten ein Vorratsgefä $B$ anbringen und eine derartige Verbindung herstellen, daß das verdampfte Wasser im selben Verhältnis aus ihm wieder ersetzt werden kann.

Aus diesem Grunde wird durch einen Niveaukonstanterhalter für eine stets gleichbleibende Spiegelhöhe gesorgt.

Die hier verwendete Niveauröhre, die durch D. R. P.-Anmeldung $23125 a$ geschützt ist, und die infolge ihrer Schmieg- und Biegsamkeit die einzig verwendbare ist, besteht aus einem beliebig gebogenen Rohre, das unten ein Loch aufweist. In der beigedruckten Skizze ist sie mit $\mathrm{N}$ bezeichnet. Wenn das Vorratsgefäß M geschlossen ist, kann Flüssigkeit nur dann in den Dampfentwicklungsraum D fließen, wenn die Öffnung der Röhre noch frei ist, so daß Luft oder Dampf nach $\mathbf{M}$ gelangen. Der Zufluß wird aber sofort aufhören, sowie das Loch durch den hochgestiegenen Flüssigkeitsspiegel verschlossen ist. Es leuchtet ein, daß auf diese Weise das Entwicklungsgefä $B$ stets mit Flüssigkeit bedeckt ist, die auf der großen Heizfläche von $D$ sehr schnell ins Sieden gerät. Um den Zufluß überhaupt za sperren, wenn das Vorratsgefäß beim Füllen durch Abschrauben von $\mathrm{S}$ geöffnet ist, dient der Hahn $\mathrm{H}$. In $\mathbf{E}$ werden die zu sterilisierenden Gegenstände eingebracht, wo sie von dem Dampf, der an den Seiten hochgestiegen ist, von oben nach unten durchdrungen werden.

Die weitere Einrichtung interessiert hier nicht: es ist das der bekannte Sterilisator, der sich in einen Trockenschrank umwandeln läßt.

Neben den Vorteilen, die der hier beschriebene Spezialfall erheischt, bietet sich noch der andere, wichtige, daB der Apparat einen fast sofortigen Beginn der Sterilisation gestattet und alle Kontrollapparate und Läutewerke, welche man sonst oft anbringt, um den Wärter zu benachrichtigen, daB von diesem Moment ab eine bestimmte Zeit innegehalten werde, können in Wegfall kommen ; schon dadurch dürfte der höhere Anschaffungspreis neutralisiert werden.

Der Sterilisator wird von der Fabrik für chemische und bakteriologische Apparate Dr. Robert Münke, Berlin, Luisenstr. 58, angefertigt.

\section{Ein Celluloseperoxyd?}

\author{
Von D. M. R. Zimmermani.
}

(Eingeg. d. 13./4. 1907.)

Mit Bezugnahme auf den Artikel von $\mathrm{Cros}$ und $\mathrm{B}$ e van über Celluloseperoxyd möchte ich bemerken, daß die Baumwolle und alle Pflanzenfasern in ähnlichem, wenn auch schwächerem Maßstabe als wie Seide und Wolle die Fähigkeit besitzen, mit gewissen ungesättigten chemischen Verbindungen in lose Bindung $\mathrm{zu}$ treten.

Nicht nur die unterchlorige Säure, sondern auch die schweflige Säure werden von der Baumwolle, hartnäckig trotz langen und sorgfältigen Spülens, zurückgehalten und können nur durch chemische Fingriffe entfernt werden. In besonders hohem Maße tritt dies ein, wenn die Baumwolle vorher mit Türkischrotöl und ähnlichen Verbindungen wie z. B. Oleïnseife behandelt wurde.

In diesen Fällen wird die unterchlorige Säure auch nicht durch anhaltendes Säuern entfernt, sondern erst durch eine Behandlung mit Bisulfit, Spülen mit Wasser und nachfolgendes Säuern wird die unterchlorige Säure beseitigt. An Stelle der Behandlung mit Bisulfit und nachfolgendem Säuern, kann auch eine Kochung mit Soda und Seife treten und führt zu dem gleichen Ziel.

Aus diesen Beobachtungen -.. aus der Praxis heraus - kann ich nicht recht an ein Celluloseperoxyd im vorliegenden Falle glauben, obwohl die Bildung eines Peroxydes, nämlich aus Wasserstoffsuperoxyd und Baumwolle - wenn auch nur vorübergehend - unter ähnlichen Bedingungen als den eben geschilderten möglich wäre. Darüber müßten direkt angestellte Versuche Entscheidung bringen.

\section{Zur Kenntnis des Chlorkalks.}

\author{
Von E. Schwarz. \\ (Eingeg. d. 27.|5. 1905.)
}

In dieser Zeitschrift (20, 754 [1907]) veröffentlicht H. D it $\mathrm{z}$ eine Kritik meines in dieser Zeitschrift (20, 138 [1907]) publizierten Aufsatzes. Der Ton der Kritik und der Mangel an experimentellen Beweisen entheben mich jeder weiterer Polemik. Ich bin leider Umstände halber außer Stande, mich derzeit mit der Chlorkalkfrage $\mathrm{zu}$ beschäftigen, und muß mir dies für eine spätere Zeit vorbehalten.

\section{Das chemische Institut der Handelshochschule Berlin.}

\author{
Von A. Bixz.
}

(Eingeg. d. 20./6. 1907.)

Die Lehrtätigkeit an einer Handelshochschule läßt es mehr als an einer anderen Hochschule empfinden, daß die Arbeit in jedem Spezialfach sich dem Ganzen anpassen muß. Denn obwohl ein Teil der Studierenden Kenntnisse in einzelnen Fächern zu erwerben sucht, gilt es doch, den Unterricht für die meisten so zu organisieren, daß ihnen die Ausbildung in den verschiedensten Disziplinen als etwas Einheitliches mitgegeben wird. 
Für jedes der Lehrfächer - Handelswissenschaften, Volkswirtschaftslehre, Jurisprudenz, Geographie, Chemie, Physik, kaufmännisches Unterrichtswesen - ergibt sich daraus die Notwendigkeit einer gewissen Beschränkung, zumal da die Studienzeit im allgemeinen nur auf vier, für die künftigenHandelslehrer auf fünf Semester bemessen ist. Andererseits soll das Wesentliche einer jeden Disziplin durchgearbeitet werden ; und außerdem wird rein wis. senschaftliche Tä. tigkeit verlangt, denn die Korporation derKaufmann. schaft hat die von ihr geschaffene Hochschule ausdrücklich dazu bestimmt, der. Lehre sowohl wie der Forschung zu dienen.

Somit erwächst für jeden Dozenten die Aufgabe, in umgrenztem Raume nach Möglichkeit das Ganze seines Faches zu bringen.

Welchen Umfang. muß unter diesen Umständen das chemische Institut einer Handelshochschule haben? Wie müssen die Räume verteilt, welches muB ihre Ausstattung sein?

Das sind Fragen, die sich beim Bau einer jeden Handelshochschule ergeben. An ihrer Beantwortung besteht zurzeit ein Interesse, wie mir mehrfache Anfragen aus dem In- und Aus. lande gezeigt haben, und aus diesem Grunde erfolgt die vorliegende Veröffentlichung. Im übrigen bezweckt dieser Aufsatz nicht, Neues zur Einrichtung chemischer Institute beizubringen. Deren gibt es so große und vollkommene $^{1}$ ), daß es sich bei dem Neubau eines kleinen

1) Vgl, E. F is c her u. M. Guth, Der Neubau des 1. chem. Instituts der Universität Berlin, 1901. - R. Anseh üt $\%$ u. R. Schulze, Das
Institutes nur darum handeln konnte, das anderen Ortes Bewährte herauszusuchen und zu kombinieren.

Das chemische Institut umfaßt den 2. und $\mathbf{3}$. Stock und einen Keller im Südflügel des Hochschulgebäudes.

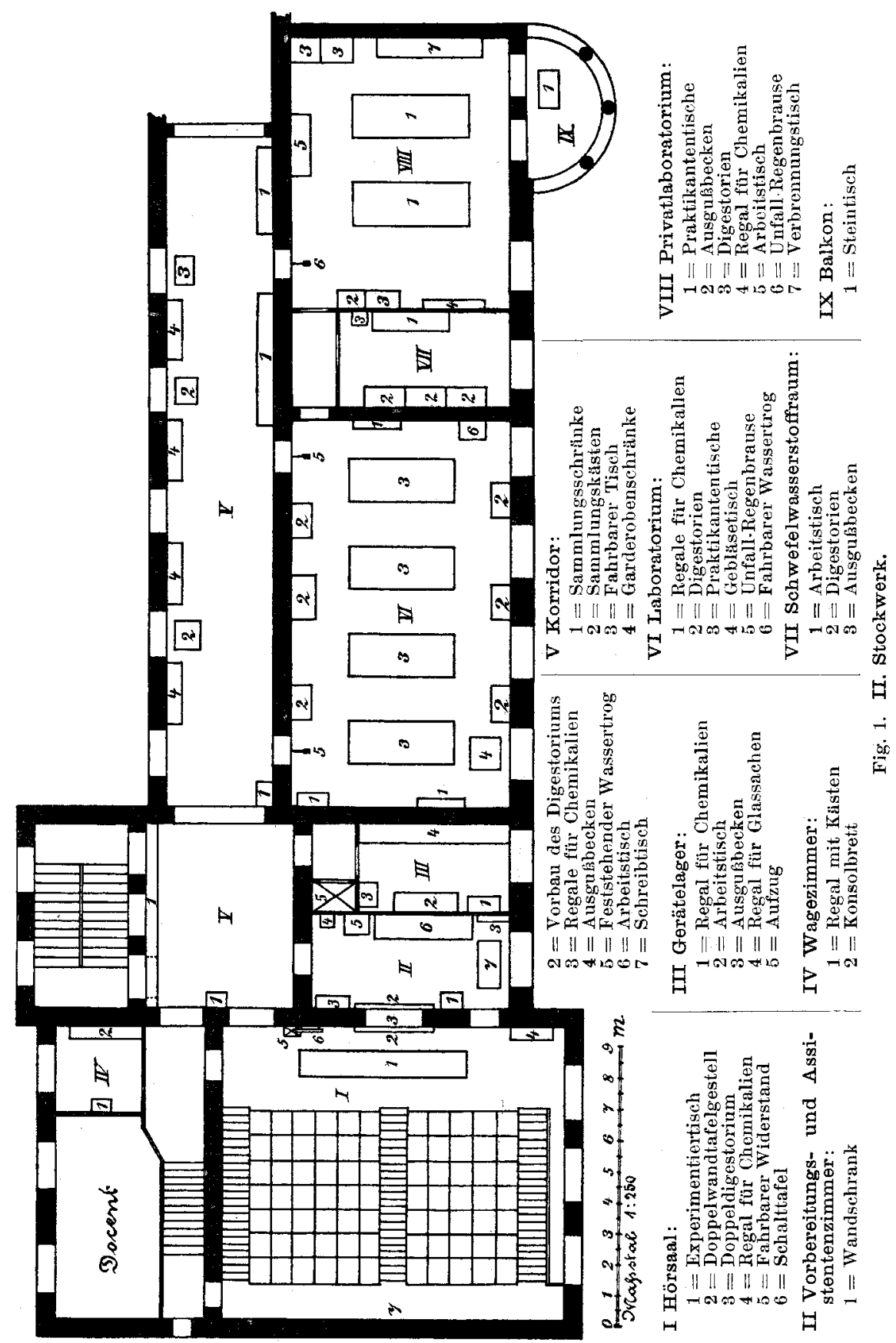

Fig. 1 zeigt das zweite Stockwerk. Der H ör $\mathrm{s}$ a a 1 hat 100 Plätze, die in üblicher Weise überein. ander angeordnet sind. Die Anzahl der Stufen einer jeden der drei Treppen im Hörsaal beträgt 14,

chem. Institut der Universität Bonn, 1904. - O. N. W it t, Das newe techn.-chem. Institut der Kgl. techn. Hochschule zu Berlin, 1906. 
ihre Höhe je $15 \mathrm{~cm}^{2}$ ). Bei diesem Grade des Ansteigens kann das, was auf dem Experimentiertisch vorgeht, von jedem Platz aus gesehen werden.

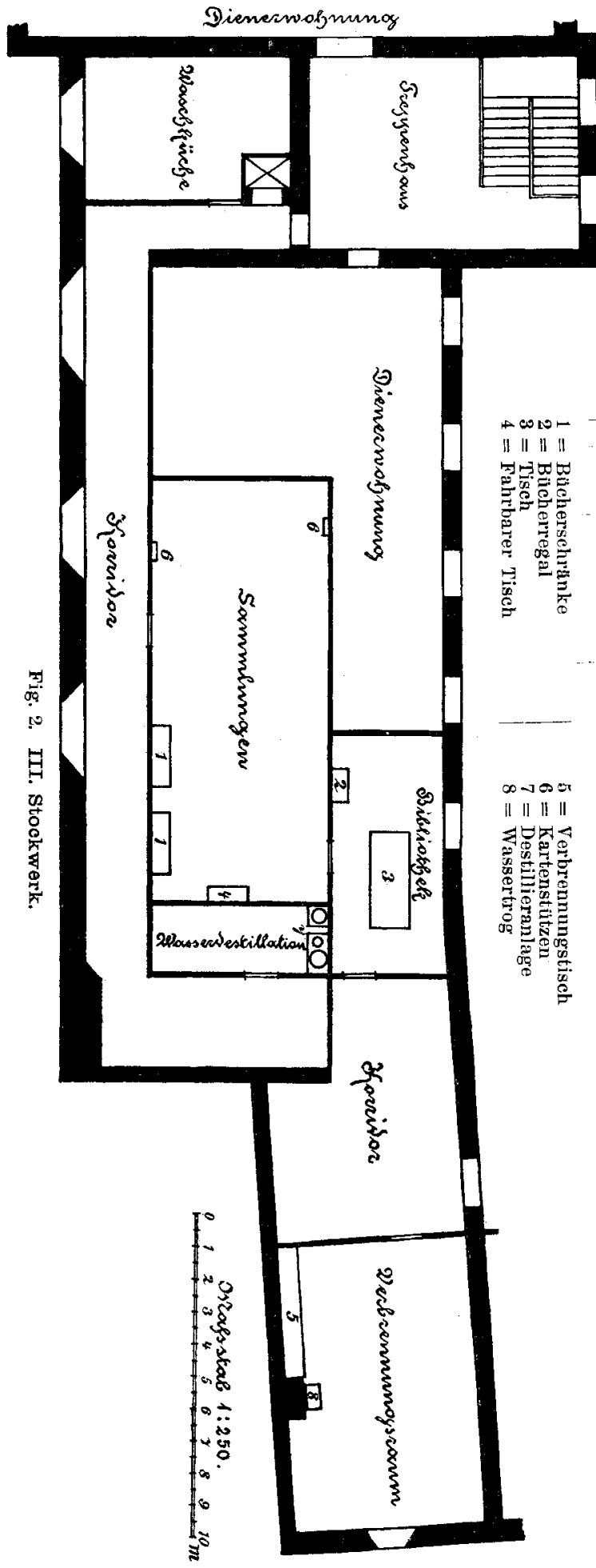

Eine stärkere Steigung erweckt meines Erachtens das Gefühl der Raumbeengung.

An der mit 7 bezeichneten Wandstelle steht

2) In der Zeichnung sind die Sitzplätze und Stufen nur schematisch angegeben. ein Projektionsapparat; der zugehörige Widerstand und die elektrische Schalttafel befinden sich rechts neben dem Vortragenden. Die Bilder werden auf eine $2,2 \mathrm{~m}$ breite und $2 \mathrm{~m}$ hohe Wandfläche über der Tafel projiziert. Durch Zug mit der Hand werden die drei Fenster von e i n e r Stelle aus verdunkelt. Ich wählte diese Einrichtung an Stelle der teureren elektrischen auf Grund der guten Erfahrungen, die im pharmazeutischen Institut der Universität Berlin damit gemacht worden sind ${ }^{3}$ ).

Der Experimentiertisch ist $5^{1 / 2} \mathrm{~m}$ lang, 0,8 $\mathrm{m}$ breit, $0,9 \mathrm{~m}$ hoch.

Das Vorbereit u n g z i m m e r dient zugleich dem Assistenten als Arbeitsraum.

Neben dem G e r ä t e 1 a $g$ e $r$ befindet sich ein Aufzug zur Verbindung mit dem Keller und dem oberen Stockwerk.

Das W a g e z i m m e r enthält ein Konsolbrett für drei Wagen und ein Regal mit verschließbaren Kästen für Gewichtssätze.

Die Sa m m l u $\mathrm{g}$ ist im Korridor untergebracht, nicht sowohl um Raum zu sparen, als um die Sammlung wie in einem Museum augenfällig auszustellen. Das wirkt wie eine Art Unterricht und empfiehlt sich meines Erachtens mehr wie die Unterbringung der Schaustücke in einem besonderen Zimmer, das erfahrungsgemäß die Studierenden nur selten betreten.

Im Praktikantensa a 1 (VI) befinden sich Arbeitstische von $3,39 \mathrm{~m}$ Länge und $1,38 \mathrm{~m}$ Breite; Abstand von Tisch zu Tisch 1,45 m. Auf jeder Seite sind 6 Schubladen und 6 Schränke. Auch die Aufsätze für die Reagenzien sind sechs. teilig. Man kann somit vorgeschrittenen Praktikanten je die Hälfte oder Anfängern je ein Drittel einer Tischseite zuweisen. Dazu kommen noch zwei Tische im Privat la boratorium. An letzteres schließt sich ein Alkoven für solche Arbeiten, die zweckmäßig im Freien ausgeführt werden.

Die A b z üg e (Digestorien) haben eine Tiefe von $0,60 \mathrm{~m}$ und $1 \mathrm{~m}$ Breite; einige größere sind $1,32 \mathrm{~m}$ breit und $0,74 \mathrm{~m}$ tief.

Das Schwefelwasserstoff $\mathrm{zimmer}$ ist durch einen Vorraum von den anstoßenden Räumen getrennt, so daß in letzteren der Geruch des Gases nicht bemerkbar wird. Den Wert dieser Einrichtung lernte ich im Institut von Herrn $O$. N. $W$ i t t kennen. Von dort übernahm ich auch die praktische Art, die Laboratorien mit destilliertem Wasser zu versorgen. Dasselbe wird im oberen Stockwerk hergestellt, fließt durch Zinnleitungen nach unten und wird aus Hähnen entnommen, die innen versilbert sind.

In sämtlichen Räumen lassen sich Schüttelapparate und Rührwerke elektrisch treiben.

Im ObergeschoB befinden sich die Wohnung des Institutsdieners, ein Reservesammlungsraum, die Wasserdestillation, eine Reserveverbrennungsraum und die Bibliothek.

Die Ausstattung des Institutes mit Apparaten trägt selbstverständlich den Bedürfnissen rein chemischer Arbeit Rechnung, auBerdem aber wurde als ein Hauptzweck im Auge behalten, daß jungen Kaufleuten eine Anschauung davon gegeben werden soll, durch welche Fäden Chemie und Warenkunde mit.

3) H. Th o m s, Privatmitteilung. 
einander verknüpft sind. Die schwierige Aufgabe, diesen Zusammenhang bei kurzer Studienzeit verständlich zu machen, ist durch die Großzügigkeit erleichtert worden, mit der die Ältesten der Kaufmannschaft die Hochschule wie im ganzen ${ }^{4}$ ) so auch in bezug auf Lehrmittel ausgestattet haben.

Von Modellen und größeren Apparaten wurden für das chemische Institut einstweilen angeschafft: eine Zeugdruckmaschine, eine Indigoküpe, der H ö m b e r g sche Apparat zum Spinnen von Kunstseide, ein Papierholländer, ein Carbidofen, die Vorrichtungen zum Bestimmen des Brennwertes der Kohle, der Festigkeit von Papier und Zement, des Flammpunktes von Petroleum.

Was der Handelshochschulunterricht für den Kaufmannsstand bedeutet, ist in einer Reihe von Schriften dargelegt worden 5 . Vom Standpunkt des Chemikers kann man hinzufügen, daß die Handelshochschulen berufen scheinen, auch die Vorbildung

4) Für Grundstück und Bau der Handelshochschule wurden über $31 / 2$ Mill. Mark ausgegeben.

5) Von neuerer Literatur sei erwähnt: J a s t r o w, Kaufmannsbildung und Hochschulbildung in Amerika, Berl. Jahrb. f. Handel u. Industrje, 1904, I, 418. - Ansprachen von Ka e mpf und von Jastrow, zur Eröffnung der Handelshochschule Berlin, bei G. R e i m er, Berlin 1906. - A p t, Zur Handelshochschul-Bewegung in Deutschland, bei C. Heymann, Berlin 1907: daselbst auch die Literatur über frühere und neuere Schriften von A p t. S c h är u. a. von Technikern in wesentlichen und bisher vernachlässigten Punkten zu erweitern. Der Chemiker, der in eine Fabrik eintritt, weiß von kaufmännischen Dingen im allgemeinen nichts. Das macht sich um so störender bemerkbar, je mehr der Neuling sich in den Betrieb einlebt und mit den Kaufleuten seiner Firma zusammenarbeiten muB, und je mehr er an der Leitung des Geschäftes teilnimmt. Hier würde es dem 'Techniker sehr zu statten kommen, hätte er einen Teil seiner Studienzeit auf einer Handelshochschule verbracht. Man lese, was dort der Lehrplan über Buchhaltung, Bilanzkunde, Kalkulationslehre und kaufmännische Arithmetik aufweist, und man wird sich des Eindruckes nicht erwehren können, daß dort ewas geboten wird, dessen der Techniker bedarf, und das er in dieser Art anderswo nicht lernen kann. Tatsächlich machen jetzt schon Chemiker und Ingenieure von der Handelshochschule Gebrauch, indem sie neben ihrem Spezialfach kaufmännische Studien betreiben.

Zum Schluß sei erwähnt, daß ich bei Anlage des Planes für die innere Einrichtung des Institutes von Herrn Ingenieur $O$. L e p p i n unterstützt wurde, dessen Firma ( $L$ e p p i n \& M a s c h e - Berlin) mit der Lieferung beauftragt war. Die Leitungen füı Gas und Wasser besorgte die Firma J. C. L. S e e I $\mathrm{m}$ e y e r - Berlin.

Meinem Kollegen Herrn F. F. M a r te n s verdanke ich die zweckmäßige Planung der elektrischen Leitungen. Mein Assistent, Herr T h. M a r x, stand mir bei der Einrichtung des Instituts mit großer Umsicht zur Seite.

\section{Referate.}

\section{I. Chemische Technologie: Apparate, Maschinen und Verfahren allgemeiner Verwendbarkeit.}

R. Rinne. Ùber das Verhalten des Magnesiumearbonats belm Kochen der Lösung. (Chem.-Ztg. 1907, 125. Aus dem Petersburger Zentrallaboratorium.)

Der Verf. kommt zur Uberzeugung, daß Magnesium in Form seines Carbonats einen sehr wesentlichen Bestandteil der bleibenden $\mathrm{H}$ ärte bilden kann, da es in geringerem Grade als kohlensaurer Kalk durch Kochen gefällt wird. Aus einer wässerigen, mit $\mathrm{CO}_{2}$ behandelten $\mathrm{L}$ ösung von $\mathrm{MgO}$ fällt je nach der Kochdauer eine gewisse Menge $5 \mathrm{MgO}$, $4 \mathrm{CO}_{2}, 5 \mathrm{H}_{2} \mathrm{O}$ aus, während der Rest als Gemisch von Carbonat und Bicarbonat in Lösung bleibt. Sechsstündiges Kochen genügt noch nicht, um alles Magnesiumcarbonat zu zerlegen.

$R d$.

E. Brauer. Erfahrungen ïber Kesselsteinverhütung.

(Z. f. Dampfk. u. Maschinenbetr. 29, 393 [1906].) Verf. hat es für günstig befunden, bei einem Kesselspeisewasser, das erst viel Kesselstein absetzte, und welches er ohne analytische Daten anzugeben als ein stark alkalisches bezeichnet, täglich eine geringe Menge süßer Maische zuzugeben. Die Kesselsteinplage habe dann aufgehört. Verf. meint, die schleimige Beschaffenheit von Zucker- und Stärkelösun- gen verhindere das Ansetzen von Kesselstein (?!), und weist zugleich darauf hin, daß vor einiger Zeit von $P$. $U \mathbf{n} \mathbf{r} \mathbf{u}$ auch ein Zusatz von Kartoffelfruchtwasser aus dem Henzedämpfer oder Rübenzuckermelasse zum Kesselwasser zu gleichem Zwecke empfohlen worden sei. (Bem. d. Ref. Derartige Zusätze können aber nicht allgemein empfohlen werden, da sie unter Umständen auch säuernde Eigenschaft des Kesselwassers hervorrufen können. Sie können höchstens bei Wässern mit hoher Carbonathärte am Platze sein, aber auch dann ist noch viel Vorsicht geboten!) - $-g$.

Keinhold Neumann. Wie erhöhe ich die Wirtschaftliehkeit meiner Kesselanlage? (Tonind.-Ztg. 31, 32, 318-20. 14./3. 1907.)

Verf. erörtert eine Reihe von Gesichtspunkten, die ohne Schwierigkeit zu beobachten sind, wie richtige Bedienung des Rostes, Bemessung des Luftzutritts unter Hinweis darauf, daß eine rauchfreie Verbrennung nicht immer rationell zu sein braucht, und da $B$ namentlich bei angestrengtem Betrieb derselbe infolge verstärkten Zuges so unrationell werden kann, daß es in vielen Fällen vorteilhafter sein wird, die Anlage zu vergrößern. Für genügende Reinigung sowohl der Züge von Flugasche wie auch des Kesselinnern von Schlamm und Kesselstein ist zu sorgen und ev. die Anlage einer Wasserreinigung in Betracht $\mathrm{zu}$ ziehen. Verf. empfiehlt dringend, dauernd 